\title{
ON CERTAIN VARIATIONS OF THE HARMONIC SERIES
}

\section{PAUL ERDÖS AND IVAN NIVEN}

Consider any block of terms from the harmonic series

$$
\frac{1}{n}+\frac{1}{n+1}+\cdots+\frac{1}{n+k-1}=S(n, k) \quad(n \geqq 1, k \geqq 1) .
$$

Define the integer $k_{2}$ by the relation ${ }^{1}$

$$
S\left(n+k, k_{2}\right)<S(n, k)<S\left(n+k, k_{2}+1\right),
$$

and similarly $k_{3}, k_{4}, \cdots$ by

(2) $S\left(n+k+k_{2}, k_{3}\right)<S\left(n+k, k_{2}\right)<S\left(n+k+k_{2}, k_{3}+1\right)$,

$$
\begin{aligned}
S\left(n+k+k_{2}+k_{3}, k_{4}\right) & <S\left(n+k+k_{2}, k_{3}\right) \\
& <S\left(n+k+k_{2}+k_{3}, k_{4}+1\right),
\end{aligned}
$$

and so on. We shall study the series

$$
\begin{aligned}
S(n, k)-S\left(n+k, k_{2}\right)+ & S\left(n+k+k_{2}, k_{3}\right) \\
& -S\left(n+k+k_{2}+k_{3}, k_{4}\right)+\cdots .
\end{aligned}
$$

TheOREM. The series (4) is convergent if and only if $k=k_{2}$.

Lemma 1. $\log (1+k / n)<S(n, k)<\log (1+2 k /(2 n-1))$.

The inequality on the left arises from the usual comparison of the harmonic series with the integral of the function $1 / x$. To prove the other inequality, we note that the convexity of the function $1 / x$ implies

$$
\int_{n-1 / 2}^{n+1 / 2} \frac{d x}{x}>\frac{1}{n} \text { or } \log \frac{2 n+1}{2 n-1}>\frac{1}{n} .
$$

We replace $n$ by $n+1, n+2, \cdots, n+k-1$ in the latter inequality and add the results.

LEMMA 2. If $k=k_{2}$, then $k=k_{j}$ with $j>2$, and the series(4) converges.

We need prove only that $k=k_{3}$. Since $k>k_{3}$ is not possible, let us

Received by the editors October 8, 1944.

${ }^{1}$ It is not possible that $S(n, k)=S\left(n+k, k_{2}\right)$. For let $h$ be the unique integer in the range $\left(n, n+k+k_{2}-1\right)$ which is divisible by the highest power of 2 , say 2 r. Let $m$ be the 1.c.m. of all the odd divisors of $n, n+1, \cdots, n+k+k_{2}-1$. Then $2^{r-1} m S(n, k)$ $=2^{r-1} m S\left(n+k, k_{2}\right)$ is an equation involving $k+k_{2}-1$ integers and the one fraction $m 2^{r-1} / h$. 
assume that $k<k_{3}$, say $k_{3}=k+r$. Then by (1) and (2)

$S(n, k)<S(n+k, k+1) \quad$ and $\quad S(n+k, k)>S(n+2 k, k+r)$.

By Lemma 1 these imply

and

$$
k / n<(2 k+2) /(2 n+2 k-1)
$$

$$
2 k /(2 n+2 k-1)>(k+r) /(n+2 k),
$$

which reduce to the incompatible inequalities

$$
2 k^{2}<2 n+k \text { and } 2 k^{2}>k(2 r-1)+2 n r-r .
$$

LEMMA 3. If $k_{2}>k$, then $k_{j+1}>k_{j}$, and moreover $k_{j+1}-k_{j} \geqq k_{2}-k$.

We prove that $k_{3}-k_{2} \geqq k_{2}-k$. Suppose that $k_{2}=k+r$, but that $k_{3}<k+2 r$. Then by (1) and (2)

and

$$
S(n, k)>S(n+k, k+r)
$$

$$
S(n+k, k+r)<S(n+2 k+r, k+2 r) .
$$

Again we use Lemma 1 as in the proof of Lemma 2 to get

$$
2 k^{2}+k+r>2 n r
$$

and

$$
2 n r+k+r>2 k^{2}+2 k r+2 r^{2} .
$$

These add to give the impossible result

$$
2 k+2 r>2 k r+2 r^{2} \text {. }
$$

LEMMA 4. If $k_{2}>k$, say $k_{2}=k+r$, then there exists an such that $k_{s}>k_{s-1}+r$.

Suppose that $k_{s}=k_{s-1}+r$ for all $s$, so that $k_{s}=k+(s-1) r$. Then the inequality

$S\left(n+k+k_{2}+\cdots+k_{s}, k_{s+1}\right)<S\left(n+k+k_{2}+\cdots+k_{s+1}, k_{s+2}+1\right)$

can be written as

$$
\begin{aligned}
s\left(n+s k+\frac{s^{2}-s}{2}\right. & \cdot r, k+s r) \\
& <S\left(n+s k+k+\frac{s^{2}+s}{2} \cdot r, k+s r+r+1\right) .
\end{aligned}
$$

We apply Lemma 1 as before to reduce this to 


$$
2 n r+2 n+2 s k+s^{2} r+k>2 k s r+s^{2} r^{2}+s r^{2}+2 k^{2} .
$$

This is impossible for $r>1$, since $s^{2} r^{2}$ is the dominating term for large $s$. If $r=1$ the inequality reduces to

$$
4 n+k>2 k^{2}+s
$$

We are now in a position to prove that (4) diverges if $k<k_{2}$. Using Lemma 4 we may assume that $r=k_{2}-k$ is as large as we please. By definition we have

$$
\begin{aligned}
S\left(n+k+k_{2}+\cdots+k_{j}, k_{j+1}\right) & -S\left(n+k+k_{2}+\cdots+k_{j+1}, k_{j+2}\right) \\
& <\frac{1}{n+k+k_{2}+\cdots+k_{j+2}} .
\end{aligned}
$$

Summing these for $j=1,2, \cdots, s-1$ (where $k_{1}=k$ ), and adding

$$
S(n, k)-S\left(n+k, k_{2}\right)<1 /\left(n+k+k_{2}\right)
$$

we obtain

$$
\begin{aligned}
S(n, k)-S\left(n+k+k_{2}\right. & \left.+\cdots+k_{s}, k_{s+1}\right) \\
& <\sum_{j=2}^{s+1}\left(n+k+k_{2}+\cdots+k_{j}\right)^{-1} \\
& <\sum_{j=2}^{s+1}\left\{n+C_{j, 2} r\right\}^{-1}<\sum_{x=1}^{s}\left(n+\frac{x^{2} r}{2}\right)^{-1} \\
& <\int_{0}^{\infty}\left(n+\frac{x^{2} r}{2}\right)^{-1} d x=\frac{\pi}{(2 r n)^{1 / 2}}<\frac{1}{2 n^{1 / 2}} .
\end{aligned}
$$

Thus we have

$$
S\left(n+k+k_{2}+\cdots+k_{s}, k_{s+1}\right)>S(n, k)-1 / 2 n^{1 / 2},
$$

and we shall complete the proof by showing that the right side of this inequality, which is independent of $s$, is positive.

With $r$ sufficiently large, inequality (5) implies that $k>n^{1 / 2}$. And since $S(n, k)$ exceeds $k$ times its smallest term, we have

$$
S(n, k)>k /(k+n)>1 / 2 n^{1 / 2} \text {. }
$$

In conclusion we shall prove that if $S(n, k)$ were defined as

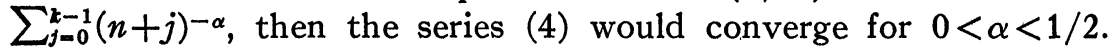
(We are unable at present to state a theorem for the cases $1 / 2 \leqq \alpha<1$.) In (1), (2), (3), and so on, the first inequality sign should be altered to include the possibility of equality. We shall prove that for $n$ sufficiently large 
(6)

$$
\begin{gathered}
\sum_{j=0}^{k-1}(n+j)^{-\alpha}<\sum_{j=k}^{2 k}(n+j)^{-\alpha} \text { or } \\
\sum_{j=0}^{k-1}\left\{(n+j)^{-\alpha}-(n+k+j)^{-\alpha}\right\}<(n+2 k)^{-\alpha},
\end{gathered}
$$

that is, that the number of terms in each block of the series is eventually a constant, which implies convergence. Since the first term of the sum on the left of the latter inequality (6) is the largest (as can be shown by elementary calculus), this inequality is implied by

$$
n^{-\alpha}-(n+k)^{-\alpha}<(n+2 k)^{-\alpha} / k \text {. }
$$

By examination of the graph of $y=x^{-\alpha}$ we see that the term on the left of the latter inequality is less than $k$ times the negative of the derivative at $x=n$, and hence we need merely prove that

$$
k \alpha n^{-\alpha-1}<\frac{1}{k}(n+2 k)^{-\alpha} \text { or } \alpha<\frac{n}{k^{2}}\left(\frac{n}{n+2 k}\right)^{\alpha} \text {. }
$$

This in turn is implied by $k^{2}<n$, as is shown by

$$
\alpha<\frac{1}{2}<\frac{n}{n+2 k}<\left(\frac{n}{n+2 k}\right)^{\alpha}<\frac{n}{k^{2}}\left(\frac{n}{n+2 k}\right)^{\alpha} .
$$

We prove that $k^{2}<n$ for $n$ sufficiently large by using the fact that every term of series (4) is less than the first one, say $c$. Thus we have

$$
k(n+k-1)^{-\alpha}<\sum_{j=0}^{k-1}(n+j)^{-\alpha}<c \quad \text { or } \quad k<c(n+k-1)^{\alpha} .
$$

When we write $k=n^{\beta}$ this becomes $n^{\beta}<c\left(n+n^{\beta}-1\right)^{\alpha}$. For $n$ sufficiently large this implies $\beta<1 / 2$, that is, $k<n^{1 / 2}$.

Purdue University 